\title{
Correction: Athletic groin pain (part 2): a prospective cohort study on the biomechanical evaluation of change of direction identifies three clusters of movement patterns
}

Franklyn-Miller A, Richter C, King E, et al. Athletic groin pain (part 2): a prospective cohort study on the biomechanical evaluation of change of direction identifies three clusters of movement patterns. Br J Sports Med 2017;51:460-8.

The authors regret that the function used to normalise joint moments to body mass in the analysis for this paper contained an error which resulted in incorrect calculation of these variables. Full re-analysis of the corrected data identified small differences from those originally reported in some phase boundaries for the relevant between-cluster comparisons, but did not affect the participant clustering or the conclusions of the study. Table 2 and Figure 5 should be replaced by the corrected versions below.

Table 2 Descriptive of the kinetic differences between clusters 1, 2 and 3

\begin{tabular}{|c|c|c|c|c|c|}
\hline Measure & Findings & Detailed & Phase & $\begin{array}{l}\text { Mean } \\
\text { difference }\end{array}$ & Effect \\
\hline \multicolumn{6}{|l|}{ Kinetics } \\
\hline \multicolumn{6}{|l|}{ GRF } \\
\hline \multirow[t]{3}{*}{$x$} & $1,2>3$ & $1>3$ & $10 \%-30 \%$ & 0.73 & 0.56 \\
\hline & & $2>3$ & $12 \%-22 \%$ & 0.82 & 0.52 \\
\hline & $3>2$ & $3>2$ & $91 \%-98 \%$ & -0.25 & 0.54 \\
\hline \multirow[t]{3}{*}{ y } & $3>1,2$ & $3>1$ & $43 \%-56 \%$ & 1.29 & 0.54 \\
\hline & & $3>2$ & $31 \%-66 \%$ & 1.87 & 0.77 \\
\hline & $2>3$ & $2>3$ & $84 \%-92 \%$ & -0.99 & 0.53 \\
\hline \multirow[t]{3}{*}{$z$} & $3>2$ & $3>2$ & $38 \%-60 \%$ & -1.98 & 0.68 \\
\hline & $1,2>3$ & $1>3$ & $76 \%-93 \%$ & 1.66 & 0.56 \\
\hline & & $2>3$ & $78 \%-98 \%$ & 1.56 & 0.64 \\
\hline \multicolumn{6}{|l|}{ Ankle } \\
\hline \multirow{4}{*}{ Plantar moment (+) } & $1,3>2$ & $1>2$ & $5 \%-78 \%$ & 0.38 & 0.71 \\
\hline & & $3>2$ & $6 \%-70 \%$ & 0.32 & 0.56 \\
\hline & $1,2>3$ & $1>3$ & $79 \%-93 \%$ & 0.04 & 0.49 \\
\hline & & $2>3$ & $82 \%-100 \%$ & 0.21 & 0.55 \\
\hline \multirow[t]{3}{*}{ Evertor moment (+) } & $1,3>2$ & $1>2$ & $14 \%-74 \%$ & 0.12 & 0.60 \\
\hline & & $3>2$ & $17 \%-73 \%$ & 0.16 & 0.73 \\
\hline & $2>3$ & $2>3$ & $82 \%-96 \%$ & 0.05 & 0.47 \\
\hline \multirow[t]{3}{*}{ Ex. rotator moment $(-)$} & $2>3$ & $2>3$ & $9 \%-23 \%$ & 0.07 & 0.49 \\
\hline & $1>3$ & $1>3$ & $86 \%-97 \%$ & 0.07 & 0.64 \\
\hline & & $2>3$ & $93 \%-100 \%$ & 0.04 & 0.45 \\
\hline \multicolumn{6}{|l|}{ Knee } \\
\hline \multirow[t]{3}{*}{ Extensor moment $(+)$} & $2>3$ & $2>3$ & $32 \%-61 \%$ & 0.45 & 0.59 \\
\hline & $1,2>3$ & $1>3$ & $72 \%-90 \%$ & 0.38 & 0.56 \\
\hline & & $2>3$ & $75 \%-89 \%$ & 0.32 & 0.46 \\
\hline \multirow[t]{7}{*}{ Valgus moment (+) } & $2,3>1$ & $2>1$ & $1 \%-7 \%$ & 0.24 & 0.72 \\
\hline & & $3>1$ & $1 \%-9 \%$ & 0.21 & 0.55 \\
\hline & $1>2,3$ & $1>2$ & $20 \%-75 \%$ & 0.43 & 0.78 \\
\hline & & $1>3$ & $17 \%-53 \%$ & 0.46 & 0.67 \\
\hline & & $1>3$ & $66 \%-83 \%$ & 0.23 & 0.50 \\
\hline & $1,2>3$ & $1>3$ & $93 \%-100 \%$ & 0.16 & 0.72 \\
\hline & & $2>3$ & $93 \%-100 \%$ & 0.14 & 0.60 \\
\hline \multirow[t]{2}{*}{ Int. rotator moment $(+)$} & $3>1,2$ & $3>1$ & $84 \%-94 \%$ & 0.05 & 0.49 \\
\hline & & $3>2$ & $91 \%-100 \%$ & 0.00 & 0.48 \\
\hline \multicolumn{6}{|l|}{ Hip } \\
\hline \multirow[t]{3}{*}{ Extensor moment $(+)$} & $2>1>3$ & $2<1$ & $31 \%-90 \%$ * & 0.47 & 0.53 \\
\hline & & $2<3$ & $9 \%-96 \%$ & 0.95 & 0.87 \\
\hline & & $1>3$ & $36 \%-92 \%$ & 0.61 & 0.67 \\
\hline \multirow[t]{2}{*}{ Adductor moment (-) } & $1,2>3$ & $1>3$ & $90 \%-100 \%$ & 0.24 & 0.55 \\
\hline & & $2>3$ & $86 \%-100 \%$ & 0.29 & 0.63 \\
\hline Int. rotator moment (+) & $1>3$ & $1>3$ & $57 \%-79 \%$ & 0.10 & 0.57 \\
\hline
\end{tabular}


Text within the results section 'Cluster 3 states Cluster three featured the predominant work done at the ankle with plantarflexor and evertor moments greater in the first part of the cycle but smaller in the latter part 75100\%.' should read 'Cluster three featured the predominant work done at the ankle with plantarflexor and evertor moments greater in the first part of the cycle but smaller in the latter part $79100 \%$.'

\begin{tabular}{|c|c|c|c|}
\hline ANKLE & $\mathrm{C}_{\ldots}^{\cdots \cdots}$ & 2 & $\underbrace{-1}_{-1+1}$ \\
\hline KNEE & $\frac{1}{-+\ldots}+=$ & $\begin{array}{l}= \\
\\
-\end{array}$ & $\mathrm{C}^{2}$ \\
\hline HIP & Ân: & Somest. & $\mathrm{A}_{-\ldots+\ldots}$ \\
\hline $\begin{array}{l}\text { CENTRE } \\
\text { OF MASS }\end{array}$ & $\left.\right|_{=1} ^{\infty}=$ & $\begin{array}{ll} & \\
& \end{array}$ & W. \\
\hline $\begin{array}{l}\text { GROUND } \\
\text { REACTION } \\
\text { FORCE }\end{array}$ & $\int_{\infty}^{\infty}$ & i) & $\frac{-2 \div=}{15}$ \\
\hline
\end{tabular}

Figure 5 Graphical representations of joint kinetics in each plane, and centre of mass and ground reaction force. Black continuous line represents cluster 1, red dotted line cluster 2 and blue broken line cluster 3. Below each graph, the shaded bars represent significant differences between the clusters.

\section{(2)}

\section{OPEN ACCESS}

Open access This is an open access article distributed in accordance with the Creative Commons Attribution Non Commercial (CC BY-NC 4.0) license, which permits others to distribute, remix, adapt, build upon this work non-

commercially, and license their derivative works on different terms, provided the original work is properly cited, appropriate credit is given, any changes made indicated, and the use is non-commercial. See: http://creativecommons.org/licenses/by-ncl 4.0\%.

(c) Author(s) (or their employer(s)) 2020. Re-use permitted under CC BY-NC. No commercial re-use. See rights and permissions. Published by BMJ.

Br J Sports Med 2020;54:e5. doi:10.1136/bjsports-2016-096050corr1

D Check for updates 